\title{
Reducing Decoherence in Fluctuation Electron Microscopy
}

Armin Zjajo ${ }^{1}$, Itai Matzkevich ${ }^{2}$, Hongchu Du ${ }^{3}$, Rafal Dunin-Borkowski ${ }^{4}$, Aram Rezikyan $^{5}$ and Michael Treacy $^{2}$

${ }^{1}$ Arizona State University, Phoenix, Arizona, United States, ${ }^{2}$ Arizona State University, United States, ${ }^{3}$ Forschungszentrum Juelich GmbH, United States, ${ }^{4}$ Forchungszentrum Jülich, Nordrhein-Westfalen, Germany, ${ }^{5}$ Corning, United States

Fluctuation Electron Microscopy (FEM) examines the scattering statistics from small volumes of thin amorphous materials in order to learn subtle details about any medium-range order (MRO) that may be present [1-4]. Both modeling and simulations show that FEM is extraordinarily sensitive to the presence of MRO, much more so than high-resolution diffraction and high-resolution imaging. The essence of FEM is to measure the 'speckliness' of diffraction (or image) data from small regions of the sample. This is achieved by determining the normalized intensity variance, which is given by,

$\mathrm{V}(\mathrm{kx}, \mathrm{ky})=\langle\mathrm{I} 2(\mathrm{kx}, \mathrm{ky})\rangle\langle\mathrm{I}(\mathrm{kx}, \mathrm{ky})>-2-1-<\mathrm{I}(\mathrm{kx}, \mathrm{ky})\rangle-1$.

$\mathrm{I}(\mathrm{kx}, \mathrm{ky})$ is the diffraction pattern intensity, and the angular brackets designate that we take the average over many different patterns, taken from different sample volumes. The last term subtracts the expected variance contribution from shot noise, where $\mathrm{I}(\mathrm{kx}, \mathrm{ky})$ intensities are the number of scattered electrons per pixel at scattering vector $(\mathrm{kx}, \mathrm{ky})$. The sensitivity of FEM to MRO is maximized when the resolution is comparable to the length scale of the MRO. Typically, this is in the $1-1.5 \mathrm{~nm}$ range.

One of the longstanding puzzles in FEM has been: "Why is the experimental normalized variance so low?" Scattering theory indicates that, with spatially-coherent illumination, the normalized variance values should be at a baseline value of around 1.0, rising above 1.0 when MRO is present. However, experimental data is always 1 to 2 orders of magnitude lower than this, despite the illumination being reasonably spatially coherent. Puzzlingly, the speckle intensity histogram, instead of being a negative exponential, as it should be for a fullycoherent experiment, follows a gamma distribution, as if the illumination had poor spatial coherence. Rezikyan et al [5] conjectured that this arises because the sample moves under the beam during data acquisition. Beam damage is one obvious contribution, but sample tilts and tympanic modes in the thin film can also contribute. Thermal diffuse scattering makes a small contribution. Transient sample charging effects may be more important than previously realized in insulating samples. All these effects add up to sample-contributed decoherence in the signal - collectively referred to as displacement decoherence.

A strategy that we are exploring, which may compensate for the slower sample motions, is to record much shorter exposures, but with commensurately more patterns. Thus, instead of taking one 1-second exposure, we take a thousand 1-millisecond exposures. The total signal is the same and, provided the camera readout noise is low, we should maintain acceptable signal-to-noise ratios. Individual patterns will have much-reduced counts and will exhibit much-increased shot noise, but there will be more patterns and the total signal is preserved. Modern electron-injection detectors have the ability to time the arrival of electrons with subnanosecond resolution, raising the prospect of improving temporal resolution.

To test the feasibility of this idea we simulated diffraction patterns from a model of 'random' tetrahedral amorphous silicon. Diffraction was from a gaussian probe of standard deviation $3.8 \AA$ (giving a nominal probe width of $1 \mathrm{~nm}$ ) that 'scanned over' many different regions, and orientations, of the model. The diffraction data was collected on a $1024 \times 1024$ array out to scattering vector $1.5 \mathrm{~nm}-1$. The variance map was calculated according to Eqn (1), with the expectation value of the (large) shot noise contribution being removed by the last term, $-\mathrm{I}(\mathrm{kx}, \mathrm{ky})-1$. Variance plots were produced by performing an azimuthal average over the radial line traces. 
It has been shown that reducing the fluence, in high signal-to-noise experiments, significantly increases the normalized variance from amorphous silicon and carbon samples [5]. However, the variance was still significantly lower than theory predicts. The simulations presented here suggest that ultra-short exposure times, with a commensurate increase in total number of patterns to compensate for the increased shot noise, could greatly reduce the decoherence arising from sample motions, or sample charging, during the data acquisition. This suggests an experimental strategy for restoring the experimental variance plot peak heights to values closer to theory, at least for sample displacement frequencies up to around $1 \mathrm{kHz}$, the detector frame rate.

Eliminating, or reducing, the variance suppression from decoherence is an important step toward the longstanding goal of inverting FEM variance and diffraction data to obtain reliable models of medium-range order in amorphous materials [6]

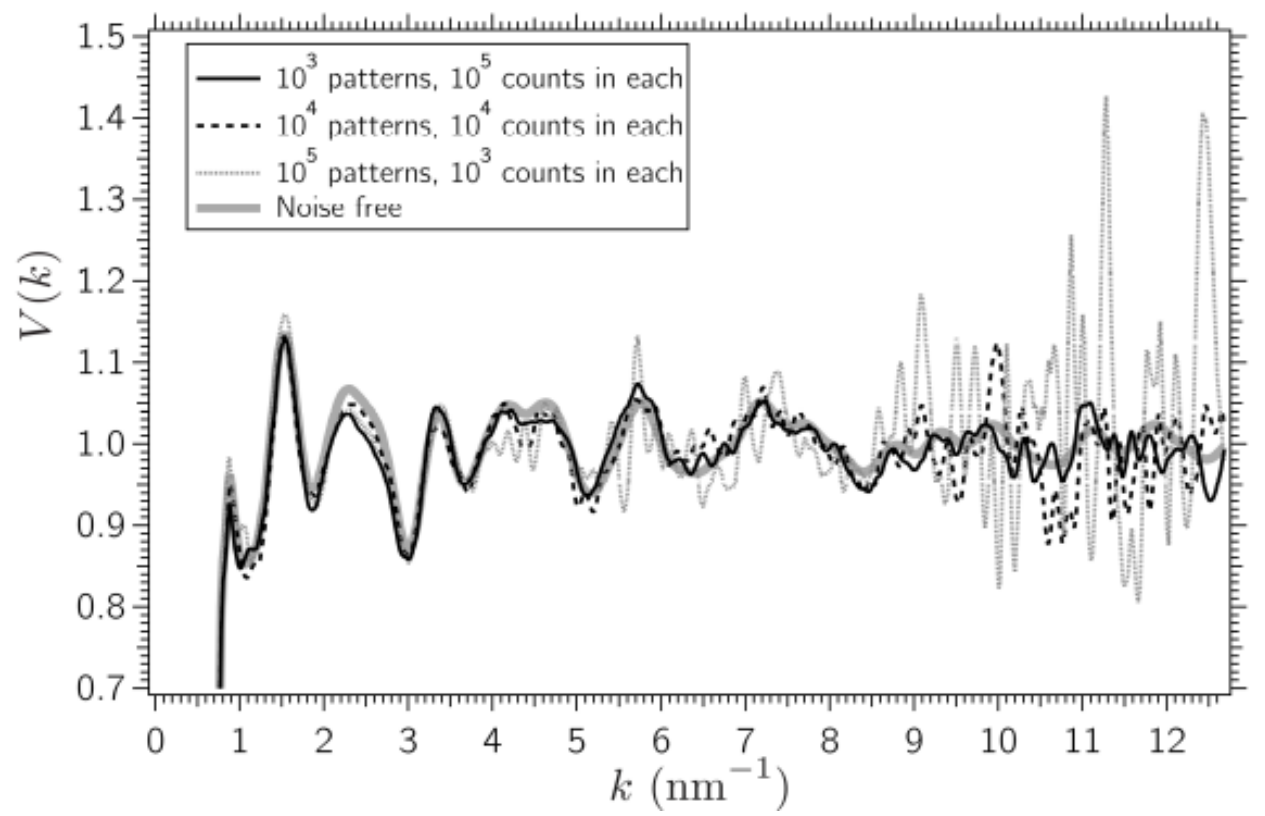

Figure 1. Kinematical variance calculations, made under high shot noise conditions, for a model of amorphous silicon with no significant medium-range order. 3 plots are made: 103 patterns each with 105 counts total; 104 patterns each with 104 counts total, and 105 patterns each with 103 counts total. The total counts in the full dataset is 108 . The variances plotted are the azimuthal averages of the $2 \mathrm{D}$ variance maps. The noise-free variance is also shown.

\section{References}

1. M.M.J. Treacy et al., Rep. Prog. Phys., 2005. 68: p. 2899-2944.

2. J.M. Gibson and M.M.J. Treacy, Phys. Rev. Lett., 1997. 78: p. 1074-1077.

3. P.M. Voyles and J.R. Abelson, Solar Energy Materials and Solar Cells, 2003. 78: p. 85-113.

4. M.M.J. Treacy et al., J. Non-Cryst. Solids, 1998. 231: p. 99-110.

5. A. Rezikyan, Z. Jibben, B.A. Rock, G. Zhao, F.A.M. Koeck, R.F. Nemanich, Microscopy and Microanalysis, 2015, 21 1455-1474.

6. Borisenko, K.B. et al, Acta Materiala, 2012, 60, 359-375. 